NTUA-102/00

hep-lat/0012028

\title{
Phase Structure of the 5D Abelian Higgs Model with Anisotropic Couplings
}

\author{
P. Dimopoulos ${ }^{(a)}$ 丹, K. Farakos ${ }^{(a)}$ ], C. P. Korthals-Altes ${ }^{(b)}$ H, G. Koutsoumbas ${ }^{(a)}$ and \\ S. Nicolis $(c)$ 团 \\ (a) Physics Department, National Technical University \\ 15780 Zografou Campus, Athens, Greece \\ (b) Centre de Physique Théorique, CNRS-Luminy \\ Case 907, F-13288 Marseille Cedex 9, France \\ (c) CNRS-Laboratoire de Mathématiques et Physique Théorique (UMR 6083) \\ Université de Tours, Parc Grandmont, 37200 Tours,France
}

\begin{abstract}
We establish the phase diagram of the five-dimensional anisotropic Abelian Higgs model by mean field techniques and Monte Carlo simulations. The anisotropy is encoded in the gauge couplings as well as in the Higgs couplings. In addition to the usual bulk phases (confining, Coulomb and Higgs) we find four-dimensional "layered" phases (3branes) at weak gauge coupling, where the layers may be in either the Coulomb or the Higgs phase, while the transverse directions are confining.
\end{abstract}

\footnotetext{
*E-mail: pdimop@central.ntua.gr

${ }^{\dagger}$ E-mail: kfarakos@central.ntua.gr

${ }^{\ddagger}$ E-mail: altes@cpt.univ-mrs.fr

$\S$ E-mail: kutsubas@central.ntua.gr

『E-mail: nicolis@celfi.phys.univ-tours.fr
} 


\section{Introduction}

The phase structure of theories with only global symmetries turns out to be insensitive to anisotropies in their couplings-e.g. the Ising model has the same critical properties on a square as well as on a rectangular lattice. For gauge theories, however, anisotropy turns out to lead to radical changes in the phase diagram. In four dimensions this implies breakdown of Lorentz invariance and is therefore physically uninteresting; for theories in higher dimensions, however, this objection no longer holds. Since all attempts towards unification involve theories defined in more than four dimensions, it is of interest to explore the phase structure of such theories in order to find four dimensional theories that are physically interesting. Until now the general approach has been a $(4+n)$ dimensional space-time with $n$ compactified dimensions; the vacuum is of the form $M^{1,3} \times X^{n}$ where $M^{1,3}$ is four-dimensional Minkowski space-time and $X^{n}$ is an internal compact space. Four-dimensional gravity exists as long as the volume of the internal space is finite. The models with non-compact internal spaces usually suffer from naked singularities. However, there are also cases where such singularities have a physical interpretation as is the case of delta-function singularities whose physical meaning invokes extended structures, such as domain walls and strings. This is the case in the Randall-Sundrum (RS) model, where the singularities may be interpreted as a four-dimensional domain wall, a three-brane, embedded in a five dimensional bulk [1]. In that case, although the internal space may be non-compact, a four-dimensional graviton exists and it is localized at the three-brane. However there are interesting subtleties to this picture that have recently been the topic of intense study [2].

The question one would now like to answer is, whether localization on the 3-brane exists for other fields, like gauge fields, fermions and scalars. In searching for localized four-dimensional fields, the equations of motion of the bulk fields which are coupled to the background geometry are solved [3]. These equations have solutions which represent localized four-dimensional massless and/or massive fields for scalars and fermions. For gauge fields things are more complicated-cf. the recent paper by Kehagias and Tamvakis in ref. [3].

In our study we wish to explore non-perturbative features, so we define our theory on the lattice and use Monte Carlo simulations to study the phase diagram of gauge fields coupled to scalars in five dimensions. In order to acquire an intuitive understanding we shall also use mean field theory. Both have already shed considerable light for the case of pure gauge theories [ [4, 5, 6, 7] as well as when fermions are included [6]. A new phase has been discovered in the pure U(1) gauge theory in 5 dimensions, where four dimensional "layers" in the Coulomb phase are separated from each other by a confining force. In particular, the transition from the five-dimensional confining phase to the layered phase turns out to be of second order [7, 8], implying the existence of new continuum theories (for suggestions in these directions cf. [9]). These features survive when fermions are introduced as well [6, 8].

In what follows we shall present evidence for the existence of a new layered phase: the layers may be in the Higgs phase, separated from each other by a confining force. A preliminary, threedimensional, version of this model has already appeared in 10 in connection with a possible application to condensed matter 2-D systems in strong magnetic fields.

The plan of the paper is as follows: In section 1 we write down the model and we recall the phase diagram for the pure $\mathrm{U}(1)$ gauge theory. In section 3 we use mean field theory to map out the phase diagram and study the order of the transitions between the different phases when scalar matter is included; in section 4 we use Monte Carlo simulations to go beyond the 
limitations of the mean field approximation and to characterize the possible phase transitions more precisely.

\section{Formulation of the model}

The model under study is the Abelian Higgs model in the five-dimensional space. Direction $\hat{5}$ will be singled out by couplings that will differ from the corresponding ones in the remaining four directions.

We proceed with writing down the lattice action of the model.

$$
\begin{aligned}
S= & \beta \sum_{x} \sum_{1 \leq \mu<\nu \leq 4}\left(1-\cos F_{\mu \nu}(x)\right)+\beta^{\prime} \sum_{x} \sum_{1 \leq \mu \leq 4}\left(1-\cos F_{\mu 5}(x)\right) \\
& +\beta_{h} \sum_{x} \operatorname{Re}\left[4 \varphi^{*}(x) \varphi(x)-\sum_{1 \leq \mu \leq 4} \varphi^{*}(x) U_{\hat{\mu}}(x) \varphi(x+\hat{\mu})\right] \\
& +\beta_{h}{ }^{\prime} \sum_{x} \operatorname{Re}\left[\varphi^{*}(x) \varphi(x)-\varphi^{*}(x) U_{\hat{5}}(x) \varphi(x+\hat{5})\right] \\
+ & \sum_{x}\left[\left(1-2 \beta_{R}-4 \beta_{h}-\beta_{h}{ }^{\prime}\right) \varphi^{*}(x) \varphi(x)+\beta_{R}\left(\varphi^{*}(x) \varphi(x)\right)^{2}\right],
\end{aligned}
$$

where

$$
\begin{gathered}
F_{\mu \nu}(x)=A_{\mu}(x)+A_{\nu}(x+\hat{\mu})-A_{\mu}(x+\hat{\nu})-A_{\nu}(x), \quad 1 \leq \mu<\nu \leq 4, \\
F_{\mu 5}(x)=A_{\mu}(x)+A_{5}(x+\hat{\mu})-A_{\mu}(x+\hat{5})-A_{5}(x) \quad 1 \leq \mu \leq 4
\end{gathered}
$$

We have allowed for different couplings in the various directions: the ones pertaining to the fifth direction are primed to distinguish them from the "space-like" couplings. The fifth direction will also be called "transverse" in the sequel.

The link variables $U_{\hat{\mu}}(x)$ are defined as $e^{\mathrm{ia}{ }_{S} \overline{\mathrm{A}}_{S}(\mathrm{x})}$ or $e^{\mathrm{ia}{ }_{\mathrm{T}} \overline{\mathrm{A}}_{\mathrm{T}}(\mathrm{x})}$ respectively, where $\bar{A}_{S}(x), \bar{A}_{T}(x)$ are the continuum fields and $a_{S}, a_{T}$ are the lattice spacings in the space-like and the transverselike dimensions respectively. The lattice fields are

$$
A_{S}(x) \equiv a_{S} \bar{A}_{S}(x), \quad A_{T}(x) \equiv a_{T} \bar{A}_{T}(x) .
$$

In addition, the scalar fields are also written in the polar form $\varphi(x)=\rho(x) e^{i \omega(x)}$. The order parameters that we will use are the following:

$$
\begin{gathered}
\text { Space - like Plaquette : } P_{S} \equiv\left\langle\frac{1}{6 N^{5}} \sum_{x} \sum_{1 \leq \mu<\nu \leq 4} \cos F_{\mu \nu}(x)\right\rangle \\
\text { Transverse }- \text { like Plaquette : } P_{T} \equiv\left\langle\frac{1}{4 N^{5}} \sum_{x} \sum_{1 \leq \mu \leq 4} \cos F_{\mu 5}(x)\right\rangle \\
\text { Space }- \text { like Link : } L_{S} \equiv\left\langle\frac{1}{4 N^{5}} \sum_{x} \sum_{1 \leq \mu \leq 4} \cos \left(\omega(x+\hat{\mu})+A_{\hat{\mu}}(x)-\omega(x)\right)\right\rangle
\end{gathered}
$$




$$
\begin{gathered}
\text { Transverse }- \text { like Link: } \quad L_{T} \equiv\left\langle\frac{1}{N^{5}} \sum_{x} \cos \left(\omega(x+\hat{5})+A_{\hat{5}}(x)-\omega(x)\right)\right\rangle \\
\text { Higgs field measure squared : } \rho^{2} \equiv \frac{1}{N^{5}} \sum_{x} \rho^{2}(x)
\end{gathered}
$$

In the above equations $N$ is the linear dimension of a symmetric $N^{5}$ lattice.

The naïve continuum limit of the lattice action (11) may be obtained as follows (where an overbar is used for the continuum fields):

$$
\begin{gathered}
\varphi=\bar{\varphi} \sqrt{\frac{2 a_{S}^{2} a_{T}}{\beta_{h}}}, \\
A_{\mu}=a_{S} \bar{A}_{\mu}, \quad 1 \leq \mu \leq 4, \\
A_{5}=a_{T} \bar{A}_{5} .
\end{gathered}
$$

Then the transverse-like field strength

$$
F_{\mu 5} \equiv A_{\mu}(x)+A_{5}(x+\hat{\mu})-A_{\mu}(x+\hat{5})-A_{5}(x) \quad(1 \leq \mu \leq 4)
$$

goes over to:

$$
-a_{S}\left[a_{T} \partial_{5} \bar{A}_{\mu}(x)\right]+a_{T}\left[a_{S} \partial_{\mu} \bar{A}_{5}(x)\right]=a_{S} a_{T}\left(\partial_{\mu} \bar{A}_{5}-\partial_{5} \bar{A}_{\mu}\right)
$$

Thus

$$
F_{\mu 5}^{2} \rightarrow a_{S}^{2} a_{T}^{2} \bar{F}_{\mu 5}^{2}, \quad 1 \leq \mu \leq 4 \quad\left(F_{\mu 5} \equiv \partial_{\mu} \bar{A}_{5}-\partial_{5} \bar{A}_{\mu}\right) .
$$

The space-like field strength is treated in a very similar way with the result:

$$
F_{\mu \nu}^{2} \rightarrow a_{S}^{4} \bar{F}_{\mu \nu}^{2}, \quad \bar{F}_{\mu \nu} \equiv \partial_{\mu} \bar{A}_{\nu}-\partial_{\nu} \bar{A}_{\mu}, \quad 1 \leq \mu<\nu \leq 4
$$

This means that the transverse-like part of the pure gauge action is rewritten in the form:

$$
\frac{1}{2} \frac{\beta^{\prime} a_{T}}{a_{S}^{2}} \sum a_{S}^{4} a_{T}\left[\sum_{1 \leq \mu \leq 4} \bar{F}_{\mu 5}^{2}\right] \rightarrow \frac{1}{2} \frac{\beta^{\prime} a_{T}}{a_{S}^{2}} \int d^{5} x\left[\sum_{1 \leq \mu \leq 4} \bar{F}_{\mu 5}^{2}\right]
$$

On the other hand the space-like part is:

$$
\frac{1}{2} \frac{\beta}{a_{T}} \sum a_{S}^{4} a_{T}\left[\sum_{1 \leq \mu<\nu \leq 4} \bar{F}_{\mu \nu}^{2}\right] \rightarrow \frac{1}{2} \frac{\beta}{a_{T}} \int d^{5} x\left[\sum_{1 \leq \mu<\nu \leq 4} \bar{F}_{\mu \nu}^{2}\right] .
$$

If we define

$$
\beta_{g} \equiv \frac{a_{T}}{g_{S}^{2}}, \quad \beta^{\prime} \equiv \frac{a_{S}^{2}}{g_{T}^{2} a_{T}},
$$

the resulting continuum action reads:

$$
\frac{1}{2} \int d^{5} x\left[\frac{1}{g_{S}^{2}} \sum_{1 \leq \mu<\nu \leq 4} \bar{F}_{\mu \nu}^{2}+\frac{1}{g_{T}^{2}} \sum_{1 \leq \mu \leq 4} \bar{F}_{\mu 5}^{2}\right]
$$


Defining $\gamma_{g} \equiv\left(\frac{\beta^{\prime}}{\beta}\right)^{1 / 2}$ and using the definitions of $\beta$, $\beta^{\prime}$ we find that

$$
\gamma_{g}=\frac{g_{S}}{g_{T}} \frac{a_{S}}{a_{T}}
$$

We denote by $\xi$ the important ratio $\frac{a_{S}}{a_{T}}$ of the two lattice spacings (the correlation anisotropy parameter) and finally derive the relation:

$$
\gamma_{g}=\sqrt{\frac{\beta^{\prime}}{\beta}}=\frac{g_{S}}{g_{T}} \xi
$$

Along the same lines, one may rewrite the scalar sector of the action in the form:

$$
\int d^{5} x\left[\sum_{1 \leq \mu \leq 4}\left|D_{\mu} \bar{\varphi}\right|^{2}+\frac{\gamma_{\varphi}^{2}}{\xi^{2}}\left|D_{5} \bar{\varphi}\right|^{2}+m^{2} \bar{\varphi}^{*} \bar{\varphi}+\lambda\left(\bar{\varphi}^{*} \bar{\varphi}\right)^{2}\right],
$$

where $D_{\mu} \equiv \partial_{\mu}-i \bar{A}_{\mu}, \quad 1 \leq \mu \leq 5$.

We have used the notations:

$$
\begin{gathered}
\gamma_{\varphi} \equiv \sqrt{\frac{\beta_{h}{ }^{\prime}}{\beta_{h}}}, \\
m^{2} a_{S}{ }^{2} \equiv \frac{2}{\beta_{h}}\left(1-2 \beta_{R}-4 \beta_{h}-\beta_{h}{ }^{\prime}\right), \quad \frac{\lambda}{a_{S}}=\frac{4 \beta_{R}}{\beta_{h}^{2} \xi} .
\end{gathered}
$$

In this paper we don't touch the problem of quantum corrections to the dependence of the space-like and transverse-like couplings on the lattice spacings $a_{S}$ and $a_{T}$, but we just consider the tree level relations derived above. Moreover, we choose a common value for the gauge coupling constants: $g_{S}=g_{T} \equiv g$, (so that $\gamma_{g}=\xi$,) and assume that all the covariant derivatives in equation (8) have the same factor in front: $\gamma_{\phi}=\xi$, in accordance with the tree-level relations. Notice that the quantum corrections give in general a $\xi$-dependence in the effective $g_{S}, g_{T}$ couplings [1]. We note that in some runs we will vary the quantities $\beta, \beta^{\prime}, \beta_{h}, \beta_{h}{ }^{\prime}$ in such a way that we have

$$
\gamma_{\varphi}=\gamma_{g} \equiv \zeta, \beta^{\prime}=\beta \zeta^{2}, \beta_{h}^{\prime}=\beta_{h} \zeta^{2}
$$

while the parameter $\beta_{R}$ is found from the equation $\beta_{R}=\frac{x \beta_{h}^{2}}{4 \beta}$, using the fixed value $x=2$ for the parameter $x \equiv \frac{\lambda}{g^{2}}$. It should be noted that our choice of parameters does not necessarily lead to an isotropic continuum theory, unless $\xi=1$.

Let us now recall the salient features of the pure $\mathrm{U}(1)$ phase diagram with anisotropic couplings (more details may be found in [4, 6, [4]). For large values for $\beta$ and $\beta^{\prime}$, the model lies in a Coulomb phase in five dimensions. There is a Coulomb force between two test charges in this phase. Now consider what will happen when one keeps $\beta$ constant, but lets $\beta^{\prime}$ take smaller and smaller values. Nothing will change in the four directions that have to do with $\beta$, so the force will still be Coulomb-like; however, the force between the test charges in the fifth direction will increase and will eventually become confining when $\beta^{\prime}$ becomes small enough. It is well known that the potential between heavy test charges is closely connected with the Wilson loops. According to the above description, the Wilson loops behave as follows: 
1. $W_{\mu \nu}\left(L_{1}, L_{2}\right) \approx \exp \left(-\sigma L_{1} L_{2}\right)$ (strong coupling) for small values of $\beta$ and $\beta^{\prime}$.

2. $W_{\mu \nu}\left(L_{1}, L_{2}\right) \approx \exp \left(-\tau\left(L_{1}+L_{2}\right)\right)$ (Coulomb phase, $\left.1 \leq \mu, \nu \leq 5\right)$, for $\beta>1$ and $\beta^{\prime}>0.4$.

3. $W_{\mu \nu}\left(L_{1}, L_{2}\right) \approx \exp \left(-\tau\left(L_{1}+L_{2}\right)\right), \quad W_{\mu 5}\left(L_{1}, L_{2}\right) \approx \exp \left(-\sigma^{\prime} L_{1} L_{2}\right)$ (layered phase, $1 \leq$ $\mu, \nu \leq 4$, ) for $\beta>1$ and $\beta^{\prime}<0.4$.

The quantities $\sigma, \tau, \sigma^{\prime}$ are positive constants. Let us remark here that there is no layered phase with the roles of $\beta$ and $\beta^{\prime}$ reversed, since the two parameters enter in a quite different way in the action. The layered phase is due to the simultaneous existence of Coulomb forces in the space-like directions and confining forces in the fifth direction.

\section{Mean Field Approach}

Our starting point is the action (1). We shall fix the gauge by imposing $U_{\hat{4}}(x)=I$ and use the translation-invariant Ansatz [4, [5, 6], $U_{\hat{\mu}}(x)=v, 1 \leq \mu \leq 3 ; U_{\hat{5}}(x)=v^{\prime}$. We also introduce the variables for the Higgs field,

$$
\phi(x)=\rho(x) v_{\chi}(x)
$$

and have also assumed a translationally invariant Ansatz, $\rho=\rho(x), v_{\chi}=v_{\chi}(x)$. The free energy, which should be minimized to get the mean field solution, reads:

$$
\begin{aligned}
F= & -3 \beta v_{a}^{2}\left(v_{a}^{2}+1\right) \\
& -\beta^{\prime} v_{a^{\prime}}^{\prime 2}\left(3 v_{a}^{2}+1\right) \\
& -\left(3 \beta_{h} v_{a}+\beta_{h}+\beta_{h}^{\prime} v_{a^{\prime}}^{\prime}\right) \rho^{2} v_{\chi}^{2} \\
& +\left(1-2 \beta_{R}\right) \rho^{2}+\beta_{R} \rho^{4}-\frac{1}{2} \log \left[\rho^{2}\right] \\
& +3 a v_{a}-3 \log \left[I_{0}(a)\right]+a^{\prime} v_{a^{\prime}}^{\prime}-\log \left[I_{0}\left(a^{\prime}\right)\right]+\chi v_{\chi}-\log \left[I_{0}(\chi)\right]
\end{aligned}
$$

The parameters $a, a^{\prime}$ and $\chi$ are conjugate to $v_{a}, v_{a^{\prime}}^{\prime}$ and $v_{\chi}$ respectively. There are three spacelike plaquettes which do not contain $U_{\hat{4}}$ and three others that do contain it; this explains the first line of expression (10). The second line contains the expressions for the transverse-like plaquettes: three of them contain $U_{\hat{4}}$ and one does not. The third line refers to the three space-like links along directions $\hat{1}, \hat{2}, \hat{3}$, the one along $\hat{4}$, and the transverse-like link. The fourth line contains terms that do not refer to directions at all; in particular the logarithmic last term comes from the measure of the Higgs field. Finally, the last line has the contributions of the integration of the Haar measure: three $a v_{a}-\log \left[I_{0}(a)\right]$ terms from the space-like links, one similar term with primed quantities from the transverse-like links and one more from the angle $\chi$ of the Higgs field.

Among the results of the minimization one may single out the relations:

$$
\left\{\begin{array}{c}
v_{a} \\
v_{a^{\prime}}^{\prime} \\
v_{\chi}
\end{array}\right\}=u\left(\left\{\begin{array}{c}
a \\
a^{\prime} \\
\chi
\end{array}\right\}\right)
$$

where

$$
u(z)=\left[\log u_{0}(z)\right]^{\prime}=\frac{u_{0}^{\prime}(z)}{u_{0}(z)}=\frac{I_{1}(z)}{I_{0}(z)}
$$


and $I_{k}(z)$ is the modified Bessel function of order $k$ :

$$
I_{k}(z)=\frac{1}{\pi} \int_{0}^{\pi} d \theta \cos (k \theta) e^{z \cos \theta}
$$

One may use these relations to eliminate variables $v_{a}, v_{a^{\prime}}^{\prime}$ and $v_{\chi}$ in favour of $a, a^{\prime}$ and $\chi$, getting an alternative form for the free energy.

We start our exploration using mean field theory sticking to the value $\beta=4.0$ for the gauge coupling within the layers, i.e. they're at weak gauge coupling. For strong gauge coupling $(\beta<1.0)$ preliminary results indicate a different picture, which will be set forth elsewhere, in order to keep the presentation clear. $x$ has been set to 2.0 and $\beta_{h}$ is running. The parameters $\beta^{\prime}$ and $\beta_{h}{ }^{\prime}$ vary according to equation (2) (the value of $\zeta$ is kept at some fixed value) and we choose $\beta_{R}=x \frac{\beta_{h}^{2}}{4 \beta}$.

A first set of results is given in figure 11. We have calculated $\rho^{2}$ for two values of $\zeta$ by mean field methods: the upper curve in the figure corresponds to $\zeta=1.0$, the isotropic model, and the lower curve to a highly anisotropic model with $\zeta=0.1$ (this means that $\beta^{\prime}=0.01 \beta, \beta_{h}{ }^{\prime}=$ $\left.0.01 \beta_{h}\right)$. The results suggest that we have Higgs transitions since $\rho^{2}$ grows large in both curves. The fact that $\rho^{2}$ does not have the tendency to increase significantly as a function of $\beta_{h}$ after some point is not hard to explain: it is due to the fact that $\beta_{R}$ is not constant, but it increases with $\beta_{h}$ according to the relation $\beta_{R}=x \frac{\beta_{h}^{2}}{4 \beta}$. The result is that $\rho^{2}$ increases as soon as the system passes to the Higgs phase and keeps increasing for not too large values of $\beta_{h}$. When $\beta_{h}$ grows to even bigger values, the resulting large value of $\beta_{R}$ forces $\rho^{2}$ to smaller values. We see that the isotropic model has the Higgs phase transition at $\beta_{h} \simeq 0.22$, while the anisotropic model at $\beta_{h} \simeq 0.27$.

We should now comment on the nature of each phase; to this end we show the behaviour of the transverse-like plaquette, $P_{T}$, in figure 2. For the isotropic model the transverse-like plaquette is the same as the space-like plaquette and it has a (fairly constant) big value. This means that all five directions of the model communicate with one another and we have a genuine five-dimensional system. In addition, $P_{T}$ has a (small) jump for $\beta_{h}=0.22$, as $\rho^{2}$ does in figure 1 (although here it is difficult to see due to the scale of the present figure) and we pass from a Coulomb to a Higgs phase. On the other hand, for the model with $\zeta=0.1$ the transverse-like plaquette is very small as compared with its space-like partner. The picture is that the model is effectively four-dimensional, since communication between the layers is very difficult, as signalled by the small values of the relevant quantities, such as $P_{T}$. Combining the conclusions drawn from figures 1 and 2 we see that for $\zeta=1.0$ we have found a transition at $\beta_{h} \simeq 0.22$ separating a five-dimensional Coulomb phase (denoted by $C_{5}$ ) from a five-dimensional Higgs phase (denoted by $H_{5}$ ). On the other hand, for $\zeta=0.1$, we have an effectively fourdimensional system, so that the transition at $\beta_{h}=0.27$ separates a four-dimensional Coulomb phase (denoted by $C_{4}$ ) from a four-dimensional Higgs phase (denoted by $H_{4}$ ). This "world" consists of four-dimensional layers, in which the symmetry is broken, connected with each other with confining forces ( $\beta^{\prime}$ is small, so we are in the confining phase of QED in this direction).

Returning to figure 1 we observe that the region between its two curves contains the curves with $0.1<\zeta<1.0$. A good means to study the behaviour of the model as a function of $\zeta$ would be to fix $\beta_{h}$ at some value and let $\zeta$ run from 0.0 to 1.0. The system will go through several phases, depending on the value of $\beta_{h}$. If $\beta_{h}<0.22$ the system will start from $C_{4}$ and at some 


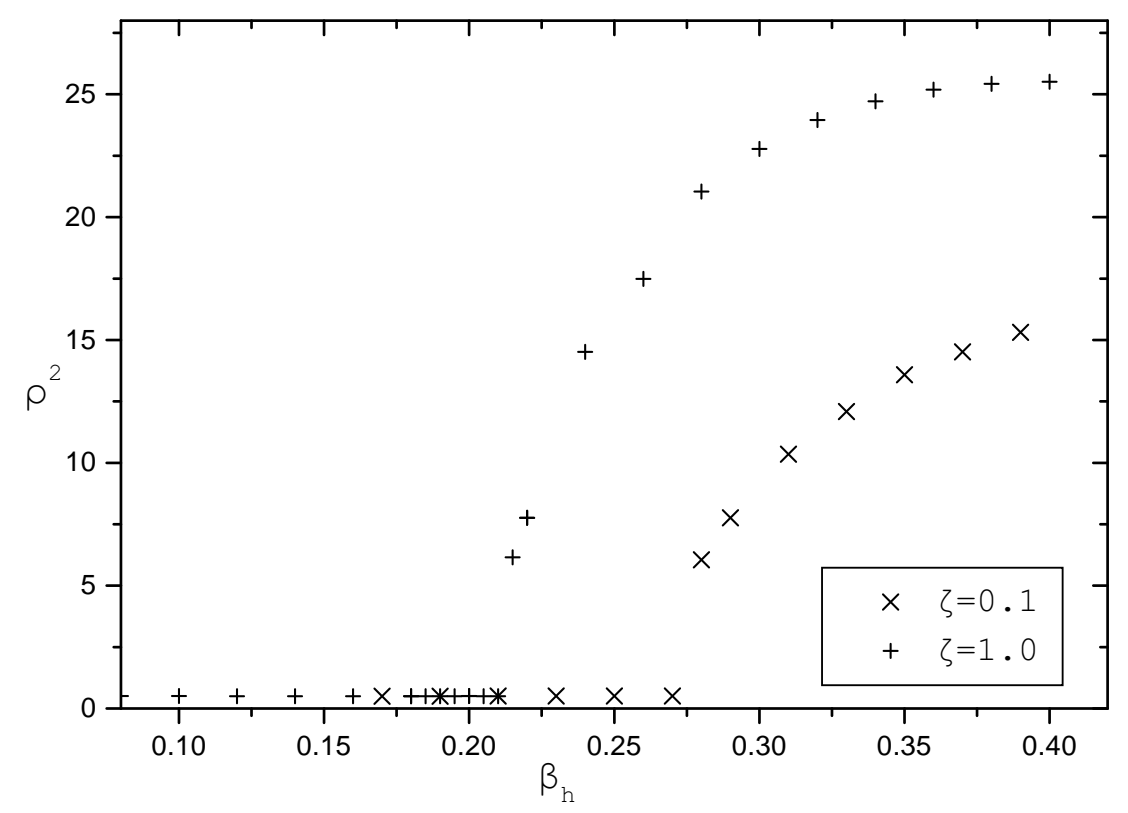

Figure 1: $\rho^{2}$ versus $\beta_{h}$ for $\zeta=1.0$ (upper curve) and $\zeta=0.1$ (lower curve) for $\beta=4.0$.

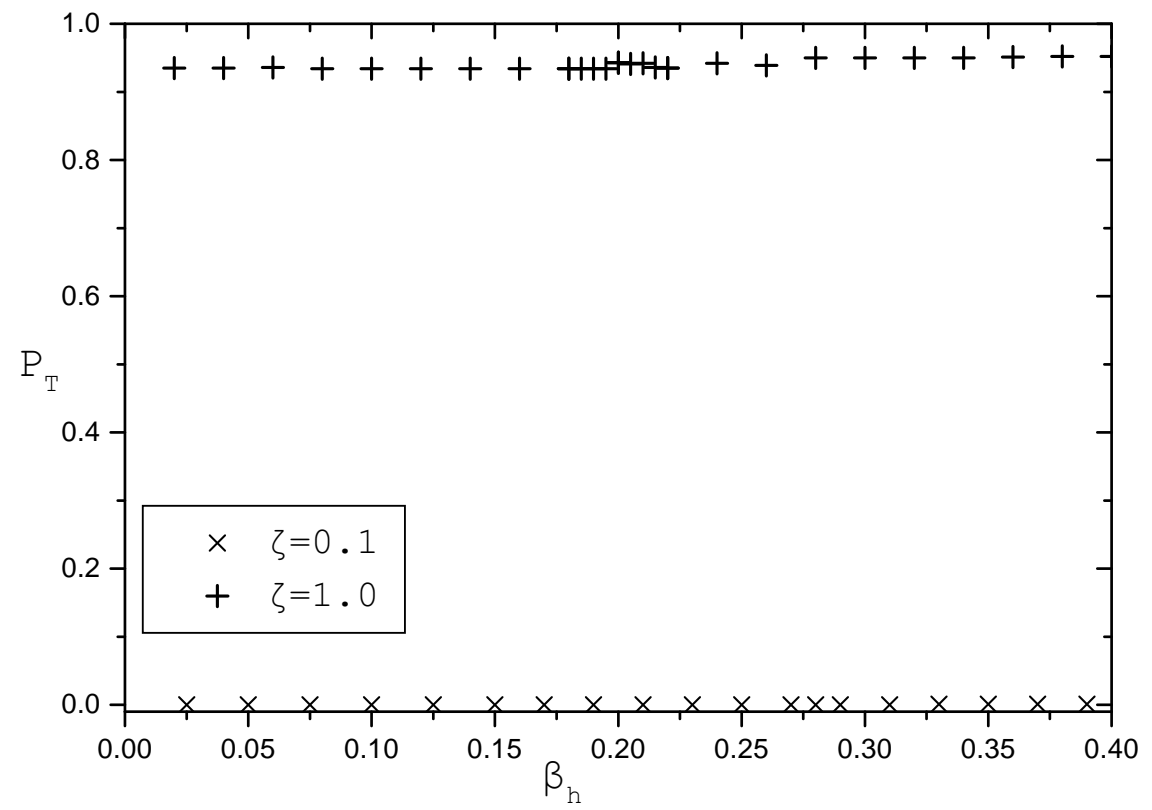

Figure 2: Transverse-like plaquette versus $\beta_{h}$ for $\zeta=0.1$ (lower curve) and $\zeta=1.0$ (upper curve) for $\beta=4.0$. 


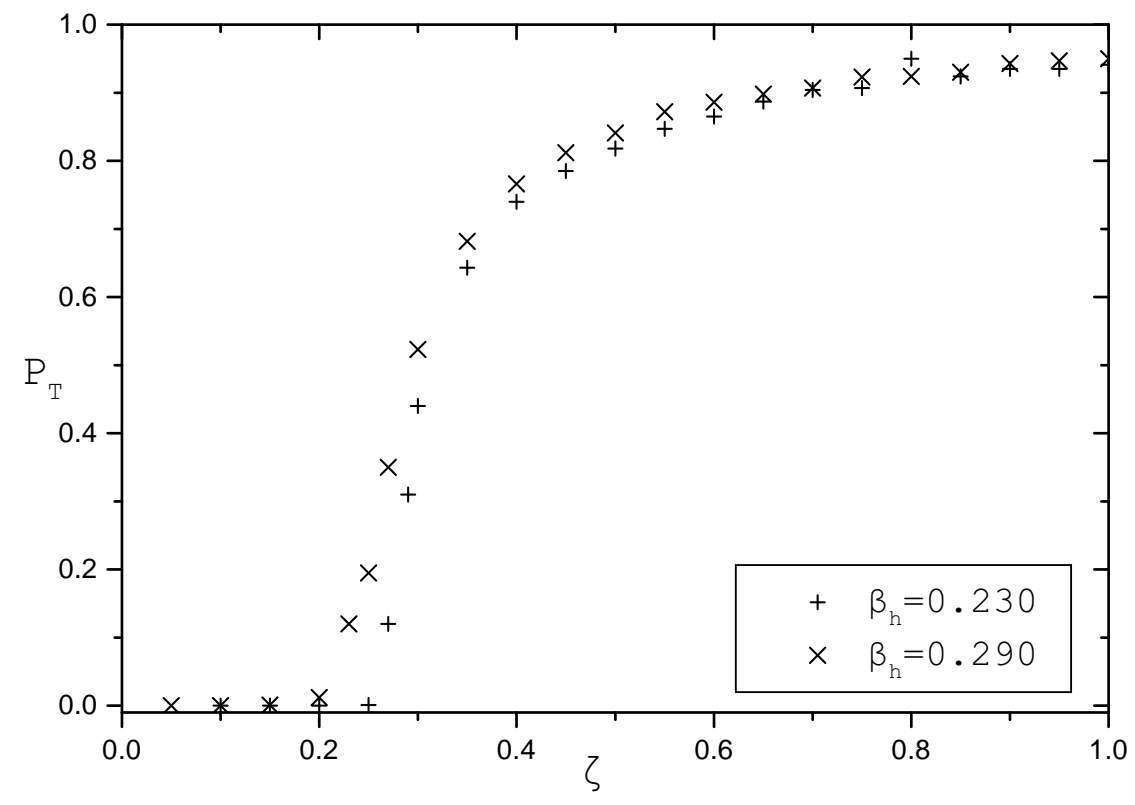

Figure 3: Transverse-like plaquette for $\beta=4.0$ and for $\beta_{h}=0.23$ (lower curve) and $\beta_{h}=0.29$ (upper curve).

point it will move to $C_{5}$. For $0.22 \leq \beta_{h} \leq 0.27$ the system will start with $C_{4}$ and will end up at $H_{5}$. It may pass through $C_{5}$ as an intermediate step or go over directly to $H_{5}$. Finally, if $\beta_{h}$ is fixed to some value bigger than 0.27 , the system will move from $H_{4}$ to $H_{5}$.

In figure 3 we have plotted the transverse-like plaquette $P_{T}$ versus $\zeta$. In the lower curve we have $\beta_{h}=0.23$, which lies in the interval $(0.22,0.27)$ refered to above. A transition is clearly visible at $\zeta \simeq 0.25: P_{T}$ is strictly zero for $\zeta<0.25$ and then grows large, so we have a fourdimensional system going over to a genuine five-dimensional one. A look at the lower curve in figure 4 , showing the corresponding variation of $\rho^{2}$ informs us that $\rho^{2}$ is small around this value of $\zeta$, signalling that the gauge symmetry is not broken at this transition. We conclude that in figure 3 we see a transition from a four-dimensional Coulomb phase $\left(C_{4}\right)$ to a five-dimensional Coulomb phase $\left(C_{5}\right)$. Another, even stronger, phase transition, takes the system into the $H_{5}$ phase. We see this in figure 4 , where $\rho^{2}$ has a discontinuity at $\zeta \simeq 0.8$.

The upper curves of figures 3 and 1 show the corresponding behaviours for $\beta_{h}=0.29$, where we expect a transition from $H_{4}$ to $H_{5}$. The transverse-like plaquette in figure 3 shows a smooth transition starting at $\zeta \simeq 0.20$. $P_{T}$ is very small for $\zeta<0.20$ but not strictly zero as happens with the $\beta_{h}=0.23$ case. This is not possible to show in a figure, so we just mention that the typical values of $P_{T}$ are of the order of $10^{-6}$ in the $\beta_{h}=0.29$ case for $\zeta<0.20$. This behaviour is consistent with the variation of $\rho^{2}$, shown in figure 4 ; its value varies from about 8 to about 22 , both of which characterize a Higgs phase. In addition, $\rho^{2}$ is constant for the interval $0<\zeta<0.20$, for which $P_{T}$ has been small.

Since we treat $\beta=4.0$ it would be useful to construct a phase diagram at $\beta=4.0$ and 


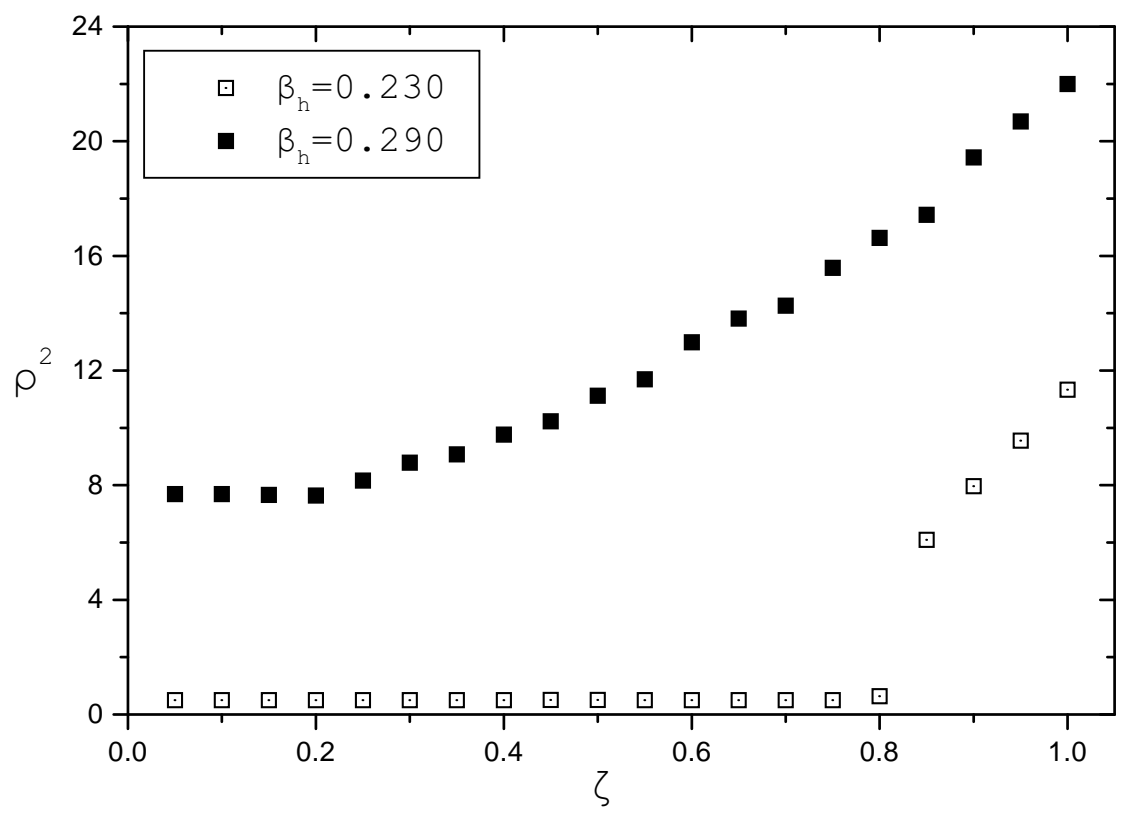

Figure 4: $\rho^{2}$ for $\beta=4.0$ and the two values $\beta_{h}=0.23$ (lower curve) and $\beta_{h}=0.29$ (upper curve).

several values of $\beta_{h}{ }^{\prime}$. The $\beta_{h}{ }^{\prime}$ coupling is one of the most important couplings, since it connects the hyperplanes in the transverse direction through the Higgs kinetic term. The diagram will be given in the $\left(\beta^{\prime}-\beta_{h}\right)$ plane. In figure 5 we show the phase diagram for $\beta_{h}{ }^{\prime}=0.001$. We observe the solid horizontal line at $\beta_{h}=0.27$, which separates the symmetric phases from the broken symmetry phases, along with the almost vertical dotted line, separating the fourdimensional phases from their five-dimensional partners. We find first order phase transitions, one separating $C_{4}$ from $H_{4}$ and another one separating $C_{5}$ from $H_{5}$. An interesting and new transition is the one from $H_{4}$ to $H_{5}$, to which we now turn.

Figure 6 shows the variation of the transverse-like plaquette versus $\beta^{\prime}$ for $\beta=4.0, \beta_{h}=$ $0.28, x=2$ and three values for $\beta_{h}^{\prime}$. For $\beta_{h}^{\prime}=0.001$ it appears that the system stays in $H_{4}$ for $\beta^{\prime}<0.24$ and then it moves very quickly to $H_{5}$. On the contrary, for $\beta_{h}^{\prime}=0.01$ the transition is smooth; it is extremely difficult to locate precisely the point of the transition. The remnant of the phase transition happens for smaller values of $\beta^{\prime}$, so if we constructed the analog of figure 5 for $\beta_{h}^{\prime}=0.01$, the dotted line would move to the left and would represent a higher order transition. For $\beta_{h}^{\prime}=0.10$ there is no region in the figure having the characteristics of $H_{4}$; the system appears to be in the $H_{5}$ phase for all $\beta^{\prime}$ couplings. The same picture emerges from figure 7, which depicts the $\beta^{\prime}$ dependence of $\rho^{2}$ rather than $P_{T}$ (we only show the results for $\beta_{h}^{\prime}=0.001$ and $\beta_{h}^{\prime}=0.01$, since the curve for $\beta_{h}^{\prime}=0.10$ lies at rather large values.) It is clear that the transition takes place at $\beta^{\prime}=0.24$ for $\beta_{h}^{\prime}=0.001$ and at a somewhat smaller value for $\beta_{h}^{\prime}=0.01$.

We have seen that there is a remarkable difference in the behaviour of the system depending 


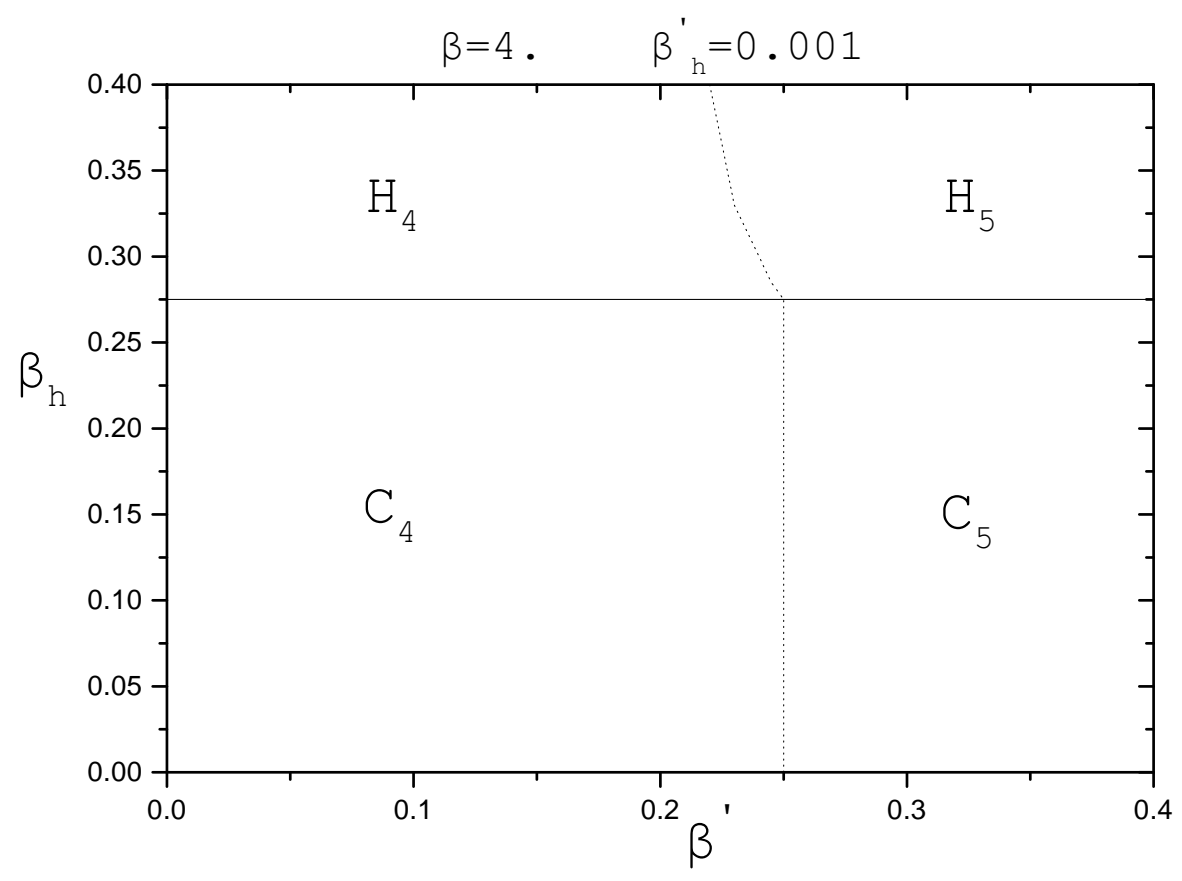

Figure 5: Phase diagram for $\beta=4.0$ and $\beta_{h}{ }^{\prime}=0.001$.

on the value of $\beta_{h}^{\prime}$, so it is natural to wonder whether there is some phase transition when $\beta_{h}^{\prime}$ varies keeping the other couplings constant. In figure 8 we display the transverse plaquette, $P_{T}$, vs. $\beta_{h}{ }^{\prime}$ for fixed values of $\beta=4.0, \beta_{h}=0.28$ and two typical values of $\beta^{\prime}(0.2$ and 0.01$)$. While the shape of the two curves seems to indicate that a step may appear for small $\beta^{\prime}$, our investigation in this range shows that a phase transition does not occur.

\section{Monte Carlo Results}

In this section we will reproduce and corroborate the Mean Field results of the previous section by Monte Carlo methods. We will find the same qualitative picture, but the critical points and the orders of the phase transitions will be determined more precisely. The sequence of the measurements will parallel the corresponding ones of the previous section.

\subsection{Fixed $\zeta$}

In this set of measurements we set $\beta=4.0, \quad x=2$, and let $\beta_{h}$ run. The remaining coupling constants depend on the value of $\zeta$, that is (we recall that $\zeta$ is the ratio: $\zeta=\sqrt{\frac{\beta^{\prime}}{\beta}}=\sqrt{\frac{\beta_{h}{ }^{\prime}}{\beta_{h}}}$ ):

$$
\beta_{h}{ }^{\prime}=\zeta^{2} \beta_{h}, \quad \beta^{\prime}=\zeta^{2} \beta, \quad \beta_{R}=\frac{x \beta_{h}^{2}}{4 \beta} .
$$




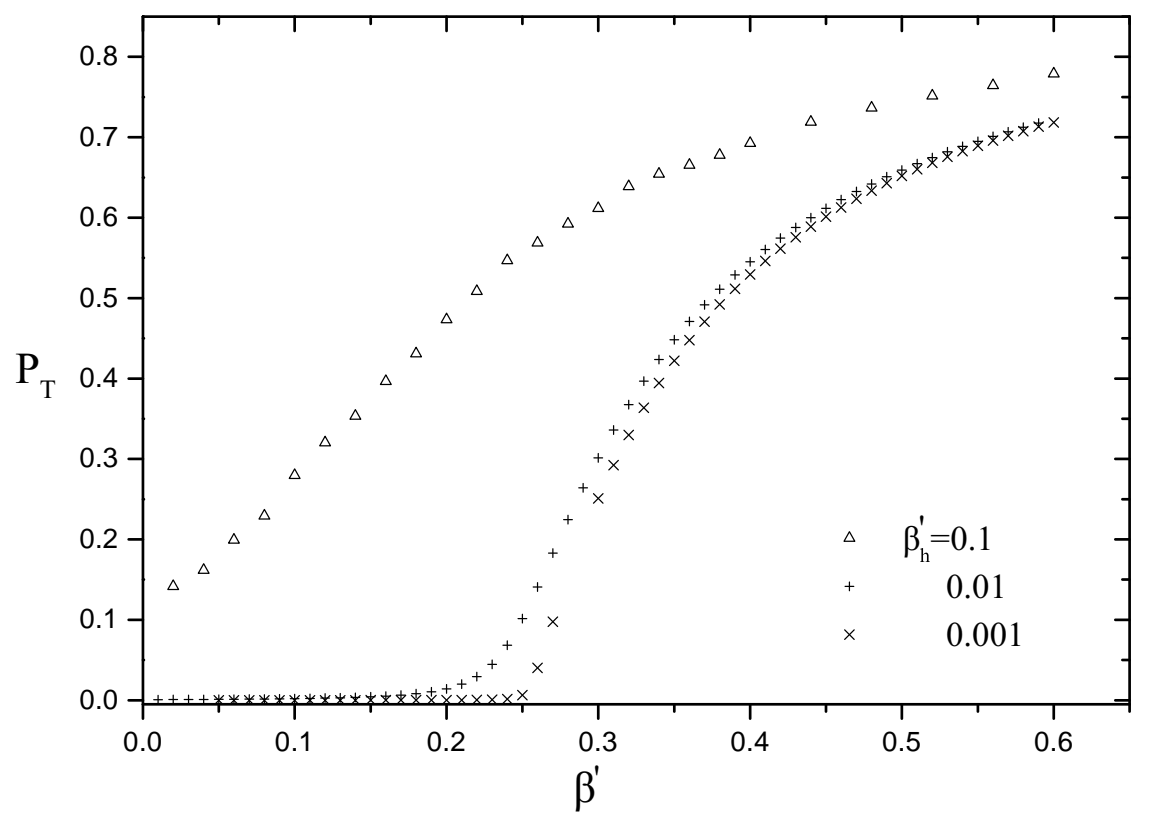

Figure 6: Transverse-like plaquette versus $\beta^{\prime}$ for $\beta=4.0, \beta_{h}=0.28$ and the values $0.001,0.01$ and 0.10 for the parameter $\beta_{h}^{\prime}$.

Thus these measurements are a Monte Carlo realization of the mean field calculations depicted in figures 11 and 2, so we expect the same qualitative picture.

In figure 9 we show the behaviour of $\rho^{2}$ for the isotropic $(\zeta=1.0)$ model. The isotropic system is seen to undergo a phase transition at $\beta_{h} \simeq 0.2075$ from the five-dimensional Coulomb phase $\left(C_{5}\right)$ to the five-dimensional Higgs phase $\left(H_{5}\right)$. We note that the critical value of $\beta_{h}$ is quite close to the mean field prediction $\left(\beta_{h} \simeq 0.22\right)$ for this quantity. The Monte Carlo result in this figure shows a jump in $\rho^{2}$, signalling a first order phase transition.

The corresponding transition for the anisotropic model $(\zeta=0.1)$ is shown in figure 10. It takes place at $\beta_{h} \approx 0.27$ and it seems smoother. In this case the "transverse-like" coupling constants are $\zeta^{2}=0.01$ times smaller than their "space-like" partners. This presumably means that the transverse-like separation $a_{T}$ of the spatial planes is much bigger than the spatial lattice spacing $a_{S}$. Support to this fact is provided by the magnitude of the quantities $P_{S}$ and $L_{S}$ that we have measured: they turn out to be much bigger than $P_{T}, L_{T}$ for the whole range of $\beta_{h}$, indicating that the quantities related to the communication of the planes are negligible as compared against the similar quantities within the layers. On the other hand (compare figure 11), $\rho^{2}$ is small in the region $\beta_{h}<0.27$, suggesting that it is a phase with unbroken symmetry. Based on these data and the mean field results, it seems safe to assume that the region $\beta_{h} \leq 0.27$ corresponds to a Coulomb phase, where the layers are decoupled: this is equivalent to the Coulomb phase of the corresponding four-dimensional model and will be called $C_{4}$ in the sequel. For $\beta_{h}>0.27$ we have a Higgs phase for the anisotropic model: $\rho^{2}$ is large and the quantities related to the fifth dimension are very small. This presumably means 


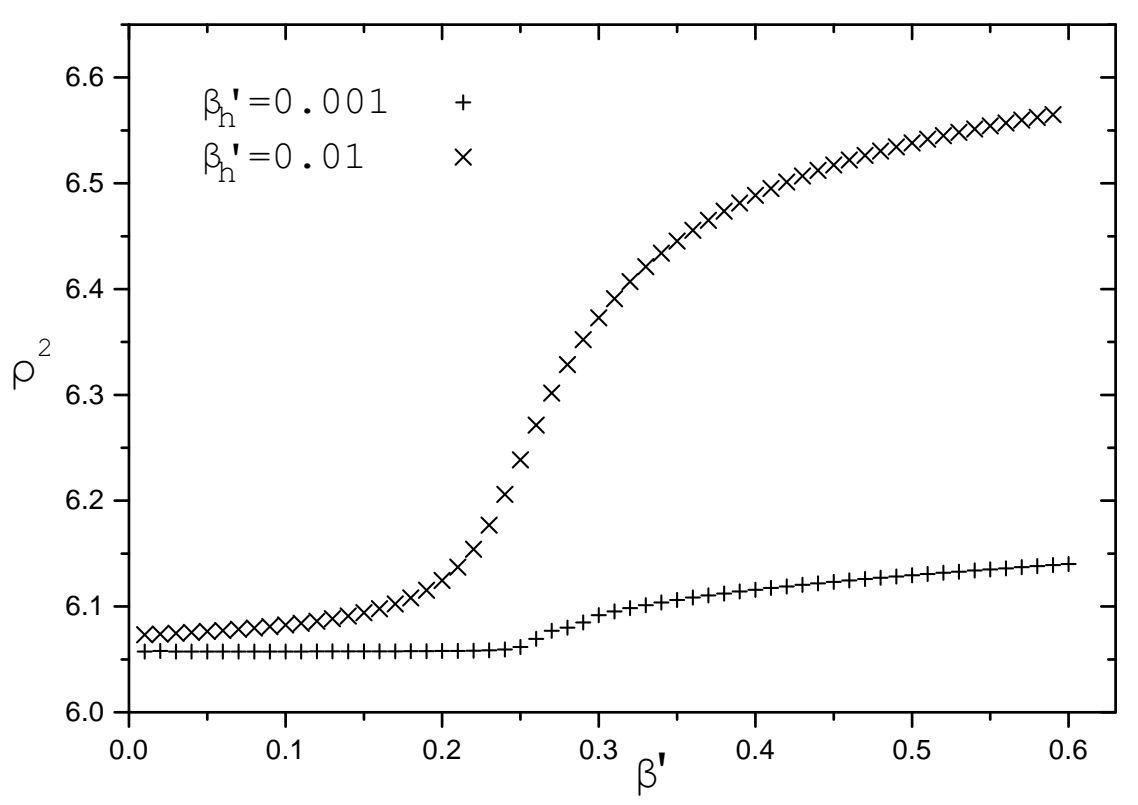

Figure 7: $\rho^{2}$ versus $\beta^{\prime}$ for $\beta=4.0, \beta_{h}=0.28$ and the values 0.001 and 0.01 for the parameter $\beta_{h}^{\prime}$.

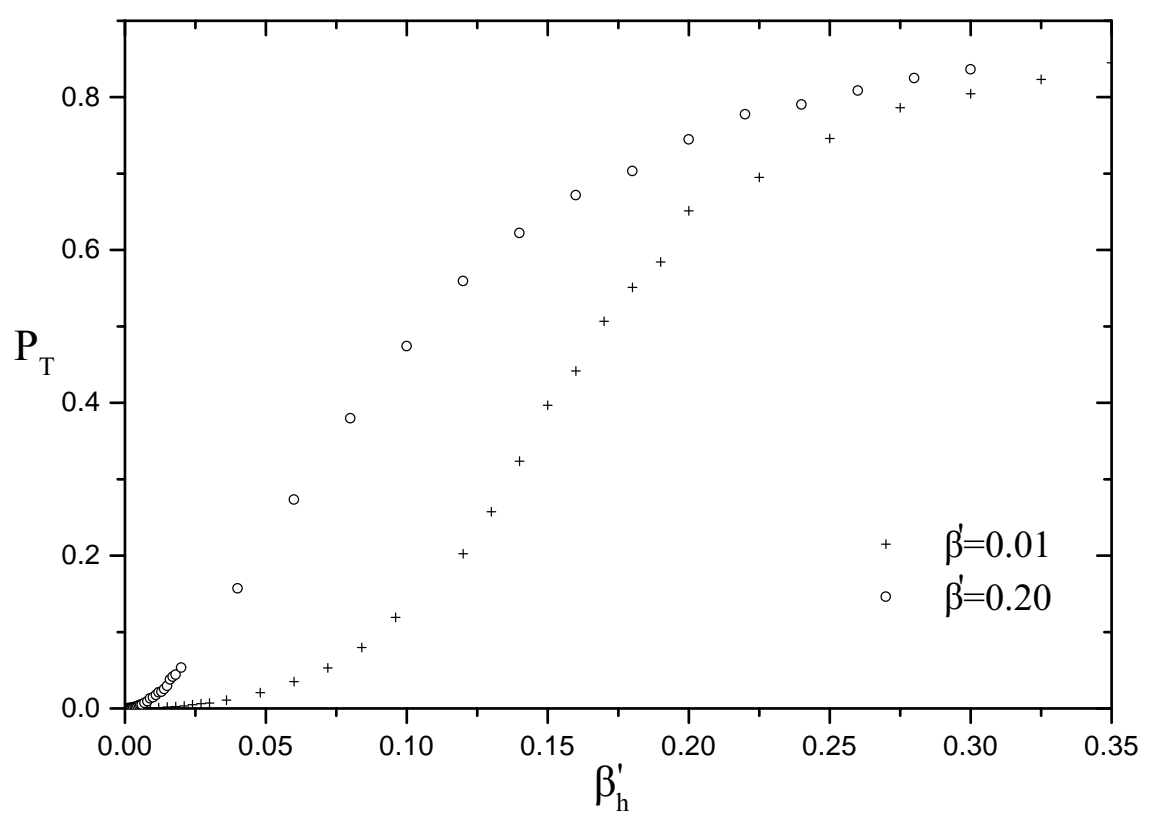

Figure 8: Transverse-like plaquette versus $\beta_{h}^{\prime}$ for $\beta=4.0, \beta_{h}=0.28$. 


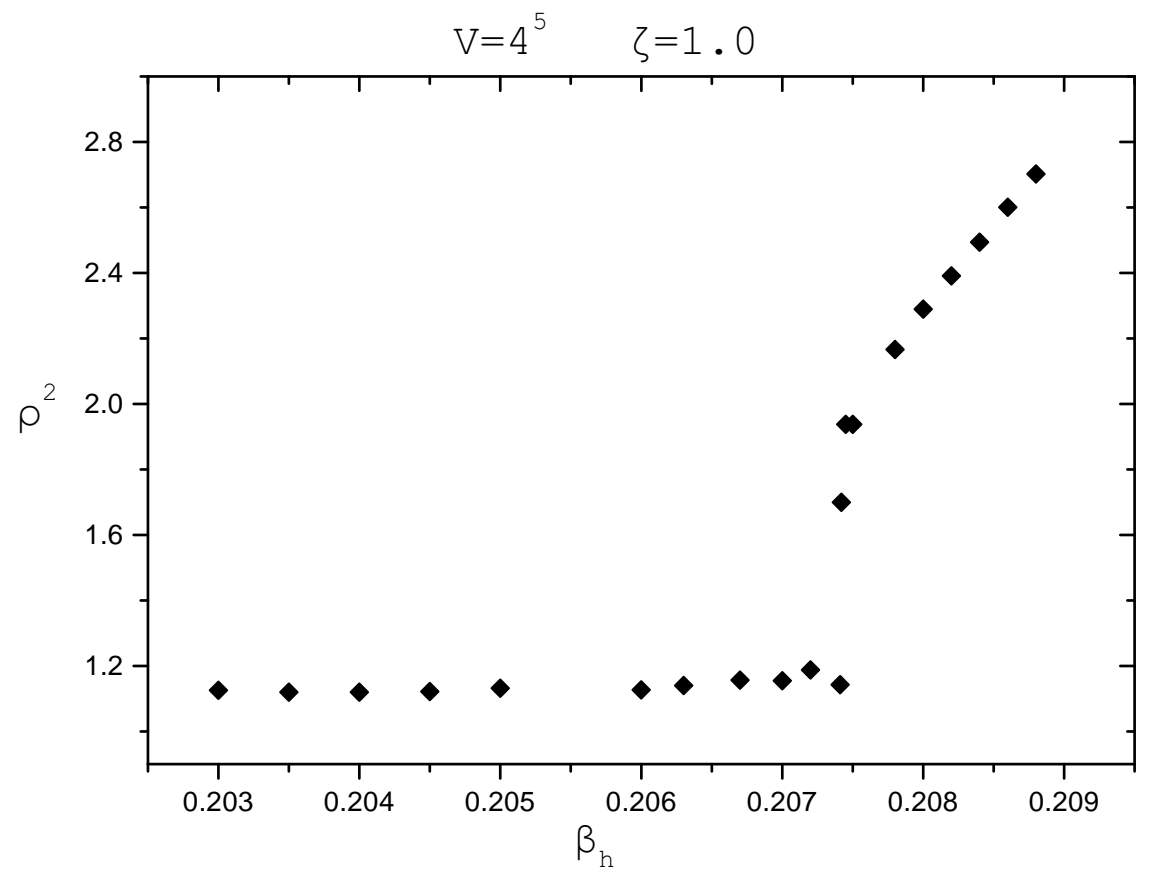

Figure 9: $\rho^{2}$ versus $\beta_{h}$ for the isotropic model $(\zeta=1.0)$ for a $4^{5}$ lattice with $\beta=4.0$.

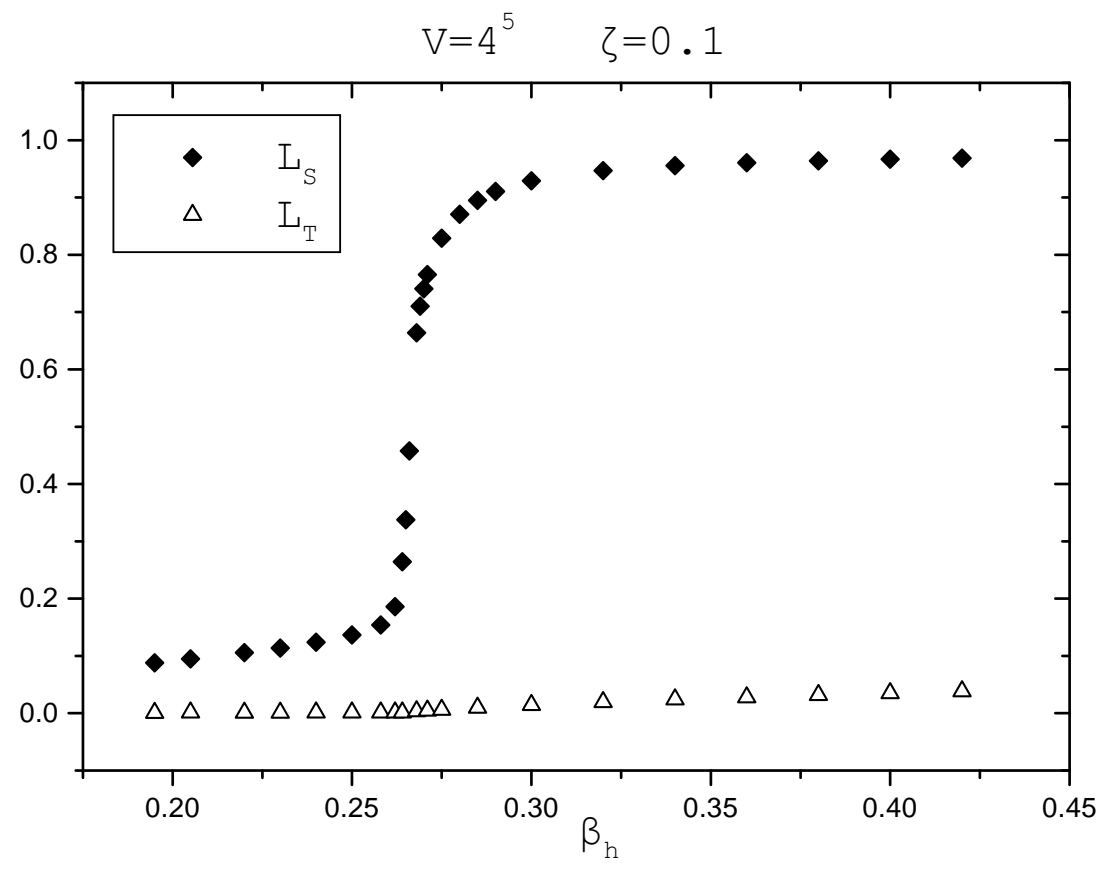

Figure 10: Space-like and transverse-like links versus $\beta_{h}$ for a highly anisotropic model $(\zeta=0.1)$ for a $4^{5}$ lattice with $\beta=4.0$. 


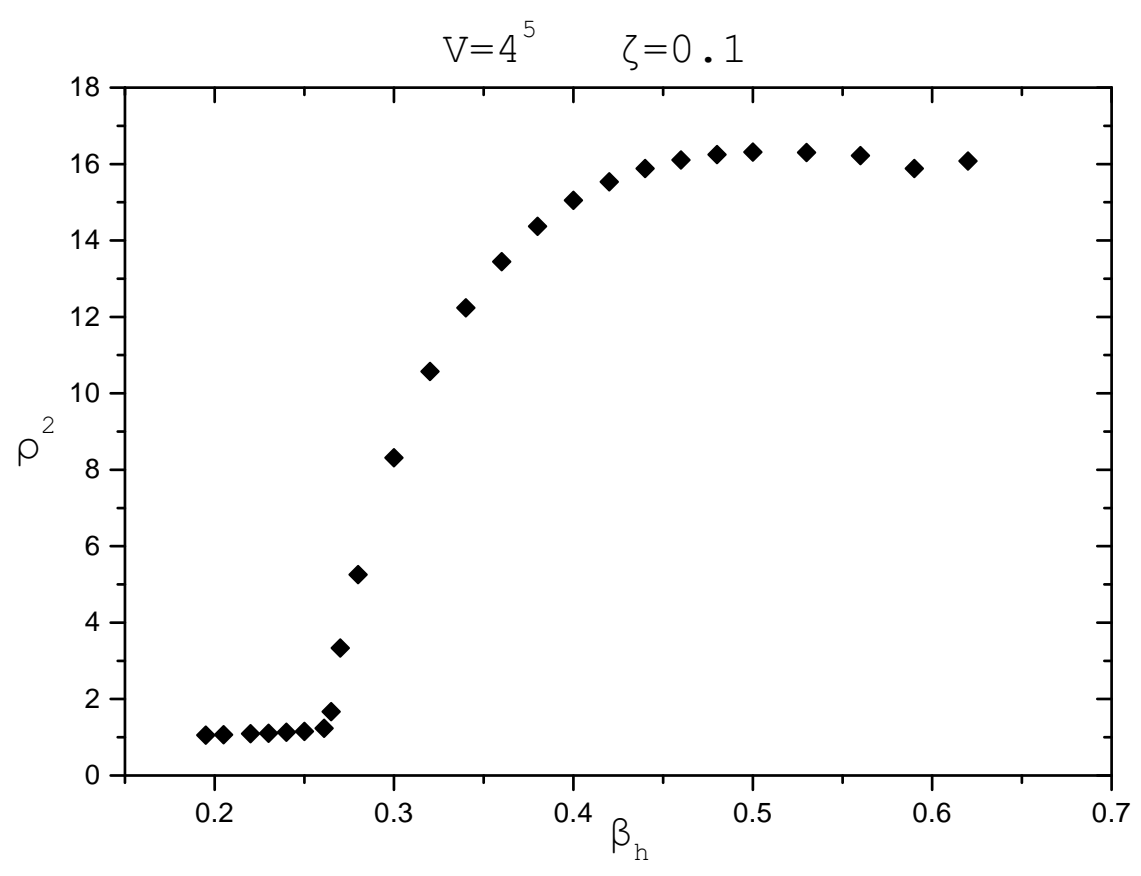

Figure 11: $\rho^{2}$ versus $\beta_{h}$ for a highly anisotropic model $(\zeta=0.1)$ for a $4^{5}$ lattice with $\beta=4.0$.

that the layers are decoupled also in this phase, so the picture is that we have moved effectively to the Higgs phase of the corresponding four-dimensional model: we will call this phase $H_{4}$. Thus in brief the anisotropic model moves from the $4 \mathrm{D}$ Coulomb phase $\left(C_{4}\right)$ to the $4 \mathrm{D}$ Higgs phase $\left(H_{4}\right)$. We remark that the critical parameter for the Higgs phase transition is of order $\frac{1}{d}$, where $d$ is the space-time dimension. Thus, it is not accidental that the isotropic model has a phase transition at $\beta_{h} \simeq 0.2075$; this is close to the expected value, $\frac{1}{d}=0.20$, since $d=5$. On the other hand, the anisotropic model is effectively four-dimensional, so one should expect a value approximately equal to $\frac{1}{4}$, which is quite close to the value 0.27 given by the simulation. A comparison with the mean field results is in order: we have found the picture predicted qualitatively by mean field theory. In particular, we found a $C_{4}-H_{4}$ transition for the anisotropic model (Monte Carlo gives it at $\beta_{h} \simeq 0.27$ and mean field also at $\beta_{h} \simeq 0.27$ ). For the isotropic model the $C_{5}-H_{5}$ transition takes place at $\beta_{h} \simeq 0.2075$ according to the Monte Carlo and at $\beta_{h} \simeq 0.22$ according to the mean field.

We note that the relative position of the transitions means that for $\beta_{h} \leq 0.2075$ the systems lie in the Coulomb phase for both models: the isotropic model in $C_{5}$ and the anisotropic one in $C_{4}$. For $\beta_{h} \geq 0.27$ the systems lie in their respective Higgs phases, $H_{5}$ and $H_{4}$. Finally, for $0.2075 \leq \beta_{h} \leq 0.27$ the anisotropic model is in $C_{4}$, while the isotropic system lies in $H_{5}$. An interesting question is how the various phases transform into one another as $\zeta$ varies from zero to one. We recall that the mean field approach suggests that the transitions take place in the order $\mathrm{C}_{4} \rightarrow \mathrm{C}_{5} \rightarrow \mathrm{H}_{5}$. A reasonable strategy would be to fix $\beta_{h}$ (as well as $\beta$ ) and vary the parameter $\zeta$; this has also been the strategy in the mean field approach. Figures 9, 10, 11 and the discussion we just made suggest that two possibly interesting values for $\beta_{h}$ would be 


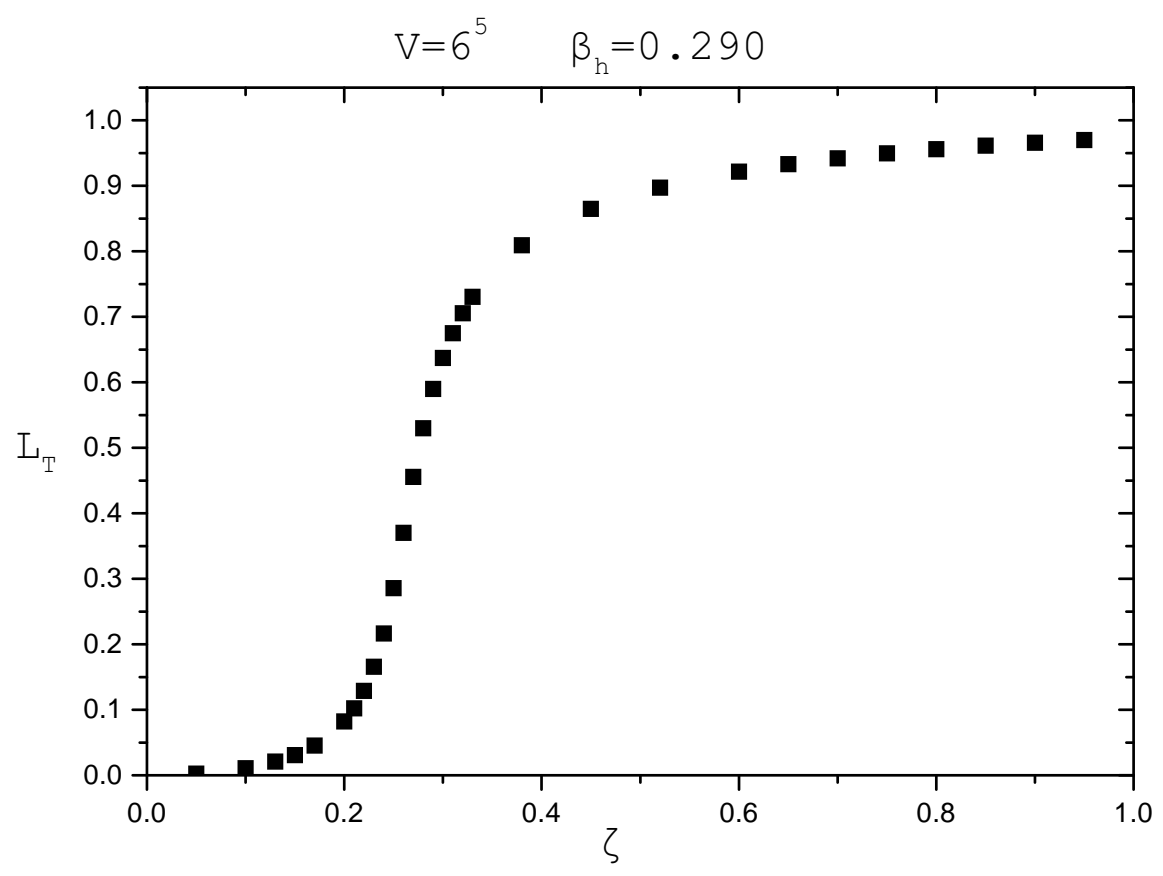

Figure 12: Transverse-like link for a $6^{5}$ lattice with $\beta=4.0, \beta_{h}=0.29$.

0.23 (to check the transition starting from the phase $C_{4}$ and ending with the phase $H_{5}$ and determine the possible intermediate steps) and 0.29 (to study the transition from $H_{4}$ to $H_{5}$ ); we point out that we had chosen exactly the same values for the mean field calculation. The Monte Carlo study at the two fixed values for $\beta_{h}$ is the subject of the following subsection.

\subsection{Running $\zeta$}

In figure 12 we show the transverse-like link $L_{T}$ for the case with $\beta_{h}=0.29$. The parameter $\zeta$ starts from zero, where the results of the previous subsection make us expect a Higgs phase with fully separated layers, that is a four-dimensional model with broken symmetry (denoted by $\left.H_{4}\right)$, to $\zeta=1.0$, where the full five-dimensional Higgs phase $\left(H_{5}\right)$ is expected on the grounds of the discussion of the previous subsection. The transition from $H_{4}$ to $H_{5}$ is very smooth and takes place at about $\zeta \simeq 0.25$. Some order parameters remain almost constant up to this value, and the ones that change, such as $L_{T}$ shown in the figure, do so very smoothly.

Figure 13 shows another aspect of the same transition, namely the gradual increase of $\rho^{2}$ versus $\zeta$; the value of this quantity is large for the whole range of $\zeta$, as would be expected for a transition from a Higgs phase to another Higgs phase. The important characteristic of this quantity is the constancy of $\rho^{2}$ for $\zeta<0.25$; the quantities $P_{S}, L_{S}$ are also constant up to this critical value of $\zeta$.

Next we examine the possibility for a non-trivial phase transition separating the $H_{4}$ and $H_{5}$ phases. In figure 14 we show the susceptibility of the transverse-like link $L_{T}$ at the $H_{4}-H_{5}$ transition for three lattice volumes, namely $4^{5}, 6^{5}$ and $8^{5}$. The data points lie on the same curve; 


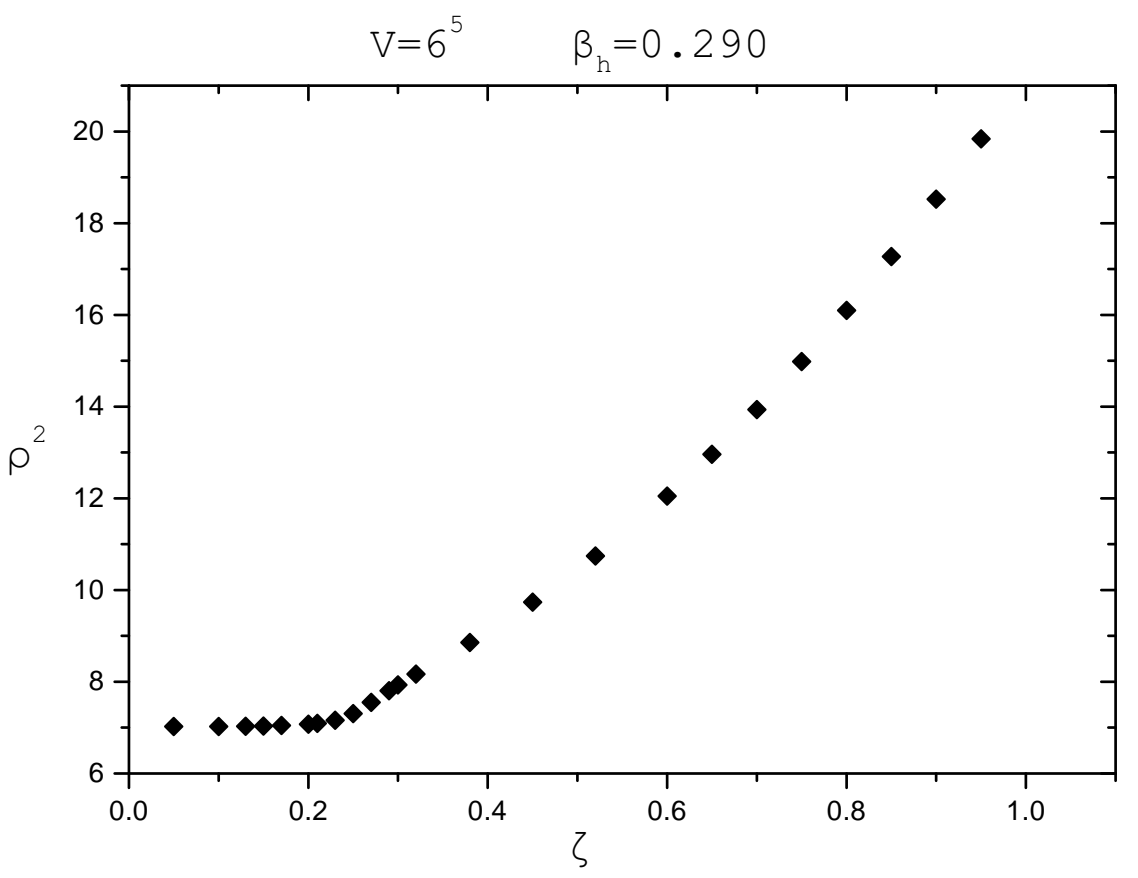

Figure 13: $\rho^{2}$ for a $6^{5}$ lattice with $\beta=4.0, \beta_{h}=0.29$.

in particular the peak is the same for the three volumes. Thus the data up to the volume $8^{5}$ suggest that this transition is a crossover.

The case where $\beta_{h}=0.23$ is more complicated. The relevant material is presented in figure 15, where the variation of the transverse-like plaquette $P_{T}$ versus $\zeta$ is shown and figure 16, which contains the variation of $\rho^{2}$. There are two phase transitions taking place as $\zeta$ increases. The first one takes place at $\zeta \simeq 0.3$, as one may see in figure 15. $P_{T}$ is tiny for small enough $\zeta$, corresponding to large anisotropy; this indicates that the layers are decoupled for these values of $\zeta$; on the other hand the values of $\rho^{2}$ turn out to be relatively small, characterizing the phase as Coulomb. Thus we are in the four-dimensional Coulomb phase, $C_{4}$. After the phase transition, the value of $P_{T}$ is sizable, so the layers communicate with each other. On the other hand, one may see in figure 16 that $\rho^{2}$ is still small at this value of $\zeta$. The result is that the system is in a five-dimensional Coulomb phase $\left(C_{5}\right)$ and the transition at $\zeta \simeq 0.3$ separates $C_{4}$ from $C_{5}$.

If we consider even bigger values of $\zeta$ we find out (as we show in figure 16) that $\rho^{2}$ jumps to big values at $\zeta \simeq 0.74$. Thus, we see the transition from the five-dimensional Coulomb phase $\left(C_{5}\right)$ to the five-dimensional Higgs phase $\left(H_{5}\right)$. Summarizing, the system moves first from $C_{4}$ to $C_{5}$ and then from $C_{5}$ to $H_{5}$, in full agreement with the mean field predictions.

The transition from $C_{5}$ to $H_{5}$ is first order. This may be seen from the distributions of the various observables. In figure 17 we show the distribution of the space-like link $L_{S}$. A clear two-peak signal is seen at $\zeta=0.737$, so we confirm a strong phase transition separating these two phases. 


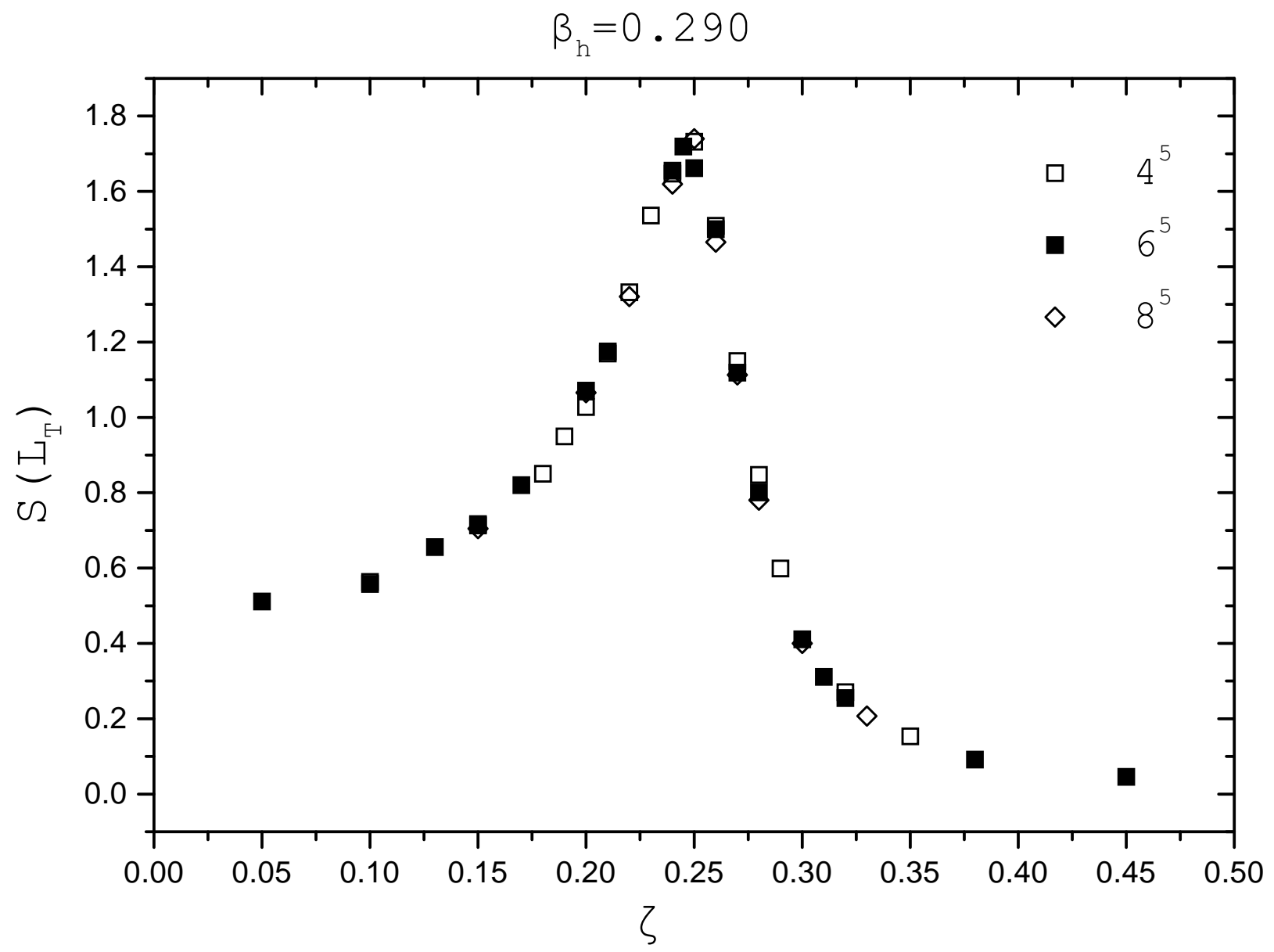

Figure 14: Susceptibility of the transverse-like link for $\beta_{h}=0.29$ and $\beta=4.0$. 


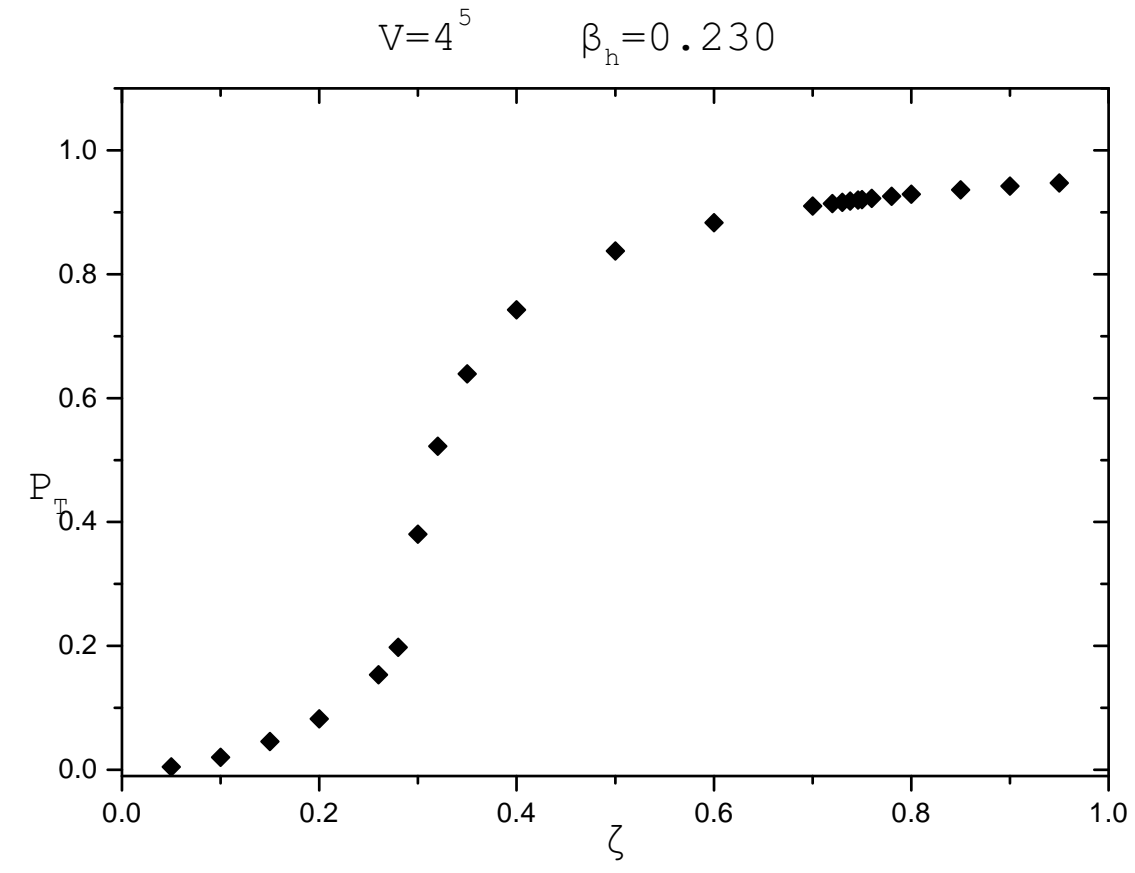

Figure 15: Transverse-like plaquette versus $\zeta$ for a $4^{5}$ lattice with $\beta=4.0, \quad \beta_{h}=0.23$.

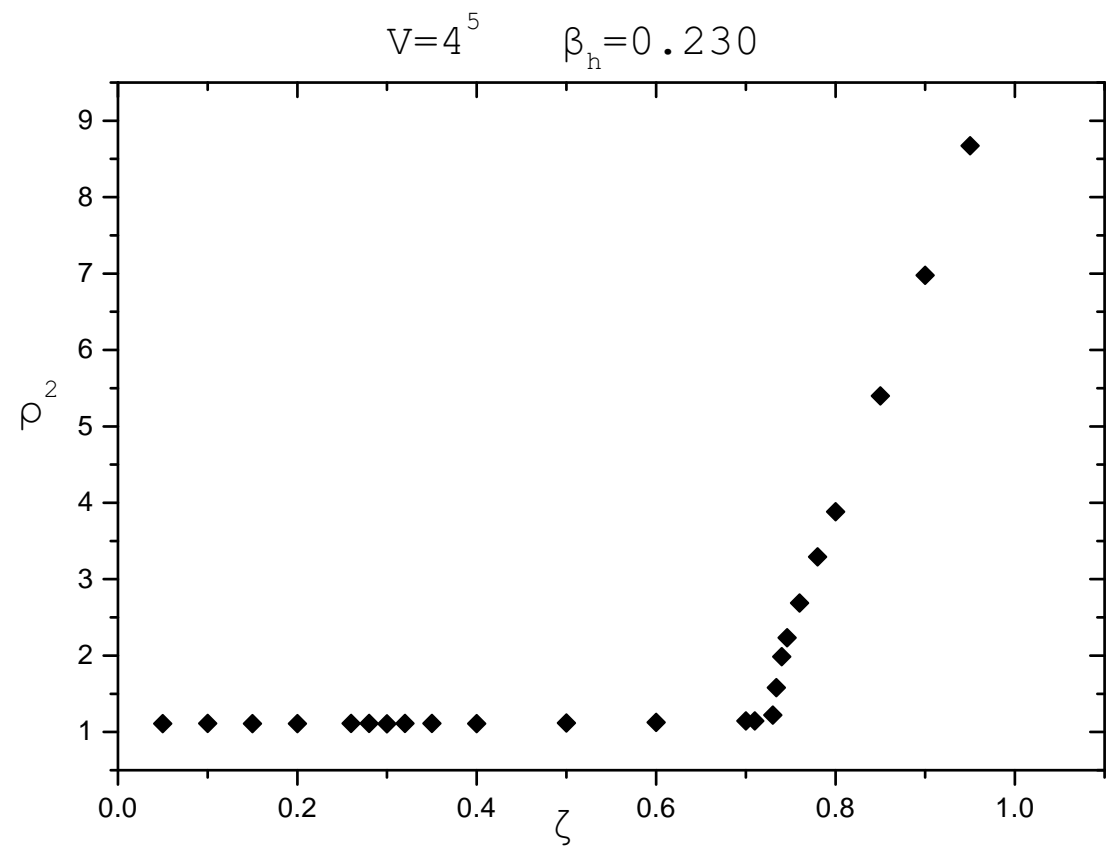

Figure 16: $\rho^{2}$ versus $\zeta$ for a $4^{5}$ lattice with $\beta=4.0, \quad \beta_{h}=0.23$. 


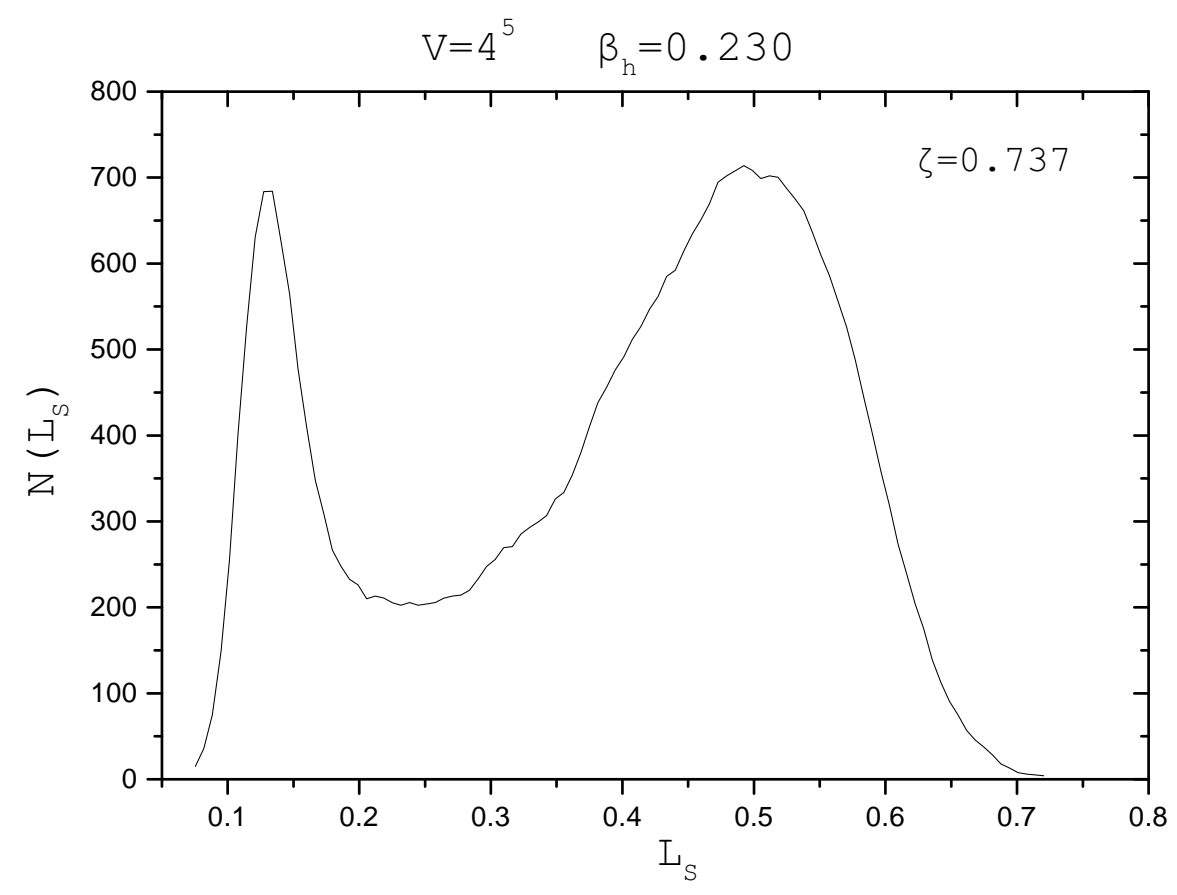

Figure 17: Distribution of the space-like link for a $4^{5}$ lattice at the $C_{5}-H_{5}$ phase transition.

\section{Conclusions}

We have explored the complicated phase structure for the Abelian Higgs model in five dimensions allowing for anisotropic couplings in the kinetic terms of the Lagrangian. This study has been done for a typical value of the (space-like) gauge coupling $\beta$ in the weak coupling regime. We search for stable four-dimensional layers (3-branes) embedded in a five-dimensional world. Besides the well known 4-D Coulomb phase $C_{4}$ (where the gauge theory is in the symmetric phase with a massless photon localized on the 3-brane) we have found indications for a new layered phase, denoted by $H_{4}$, where the gauge symmetry is broken and the 4-D properties predominate. The gauge theory is in the confining phase in the bulk. For the range of the quartic couplings that we used, $C_{4}$ and $H_{4}$ are separated by a first order transition. The $H_{4}$ and $H_{5}$ (four- and five-dimensional Higgs) phases are separated by a crossover up to the lattice volume we have studied, so they appear to be analytically connected; the realization of a continuum four-dimensional world within a five-dimensional continuum strongly depends on the parameters of the theory. The Monte Carlo results confirm qualitatively the predictions we got from the mean field calculations; the precise characterization of the phase transitions comes exclusively from the Monte Carlo approach.

Acknowledgements: P. D., K. F. and G. K. acknowledge support from the TMR project "Finite temperature Phase Transitions in Particle Physics", EU contract FMRX-CT97-0122. S. N. acknowledges the warm hospitality of the High Energy Theory Group at the National Technical University of Athens. 


\section{References}

[1] L.Randall and R.Sundrum,Phys. Rev. Lett. 83 (1999) 4690, hep-th/9906064;Phys. Rev. Lett.83(1999)3370, [hep-ph/9905221] ; A. Karch and L. Randall, "Linearly Localized Gravity", [hep-th/0011156]; A. Davidson and P. D. Mannheim, "Dynamical Localization of Gravity", [hep-th/0009064];

[2] G. Dvali, G. Gabadadze and M. Porrati, Phys. Lett. B484 (2000) 112, [hep-th/0002190]; Phys. Lett. B484 (2000) 129, [hep-th/0003054]; Phys. Lett. B485 (2000) 208, [hep-th/0005016].

[3] W. D. Goldberger and M. B. Wise, Phys.Rev.Lett. 83, 4922 (1999), [hep-ph/9907447]; Phys.Rev. D60, 107505 (1999), [hep-ph/9907218]; H. Davoudiasl, J. L. Hewett and T. G. Rizzo, Phys.Lett. B473, 43 (2000), [hep-ph/9911262]; T. Gherghetta and A. Pomarol, Nucl.Phys. B586 (2000) 141, [hep-ph/0003129]; A. Pomarol, Phys.Lett. B486 (2000)153, hhep-ph/9911294]; O. DeWolfe, D. Z. Freedman, S. S. Gubser and A. Karch, Phys. Rev. D62 (2000) 046008, [hep-th/9909134] A. Kehagias and K. Tamvakis, "Localized Gravitons, Gauge Bosons and Chiral Fermions in Smooth Spaces generated by a Bounce", [hep-th/0010112].

[4] Y. K. Fu and H. B. Nielsen, Nucl. Phys. B236 (1984) 167.

[5] A. Lowe, Z. Phys. C34 (1987) 243.

[6] C. P. Korthals-Altes, S. Nicolis and J. Prades, Phys. Lett. B316 (1993) 339, [hep-lat/9306017]; A. Hulsebos, C. P. Korthals-Altes and S. Nicolis, Nucl. Phys. B450 (1995) 437, [hep-th/9406003].

[7] P. Dimopoulos, K. Farakos, A. Kehagias and G. Koutsoumbas, "Lattice Evidence for Gauge Field Localization on a Brane", [hep-th/0007079].

[8] A. Hulsebos, Nucl. Phys. (Proc. Suppl.) 42 (1995) 618, [hep-lat/9412031].

[9] J. Ambjørn, D. Espriu and N. Sasakura,Mod. Phys. Lett. A12 (1997) 2665, [hep-th/9707095].

[10] P. Dimopoulos, K. Farakos and G. Koutsoumbas, "Decoupling of Layers in the Threedimensional Abelian Higgs Model", [hep-lat/0005012], Phys. Rev. D63 (2001) in press.

[11] F.Karsch, Nucl. Phys. B205 [FS5](1982) 285; J.Engels, F.Karsch, H.Satz, I.Montvay, Nucl.Phys. B205 [FS5] (1982) 545. 